\title{
Proposals for Future Studies in Genetics
}

\author{
Posthumous Publication of Notes by \\ Dr. S. G. SPICKETT, B.SC., PH.D. \\ Department of Genetics, University of Cambridge
}

\begin{abstract}
(With the help of Professor Thoday of Cambridge and Dr. S. G. Browne of the Leprosy Study Centre, 57a Wimpole Street, London, W.1, we have discovered these notes which contain a draft of Dr. Spickett's plans for a study of genetics and leprosy. Dr. Spickett had completed Parts I and II and they were published in Leprosy Review, 33, 1962, and as we may never find the original script of Parts III and IV we have decided to publish this draft, considering that it will be of value to other geneticists who wish to study this important subject in leprosy and as a memorial to his name.)
\end{abstract}

The suspicion that leprosy is familial has been held since ancient times, and there can be little doubt that the probability of an individual contracting the disease is much higher if there is a member of the family with leprosy than if there is no member of the family so afflicted. It is probable, however, that the familial factor in the epidemiology of leprosy is complex, and that if there is any genetic effect it is but a component of the familial effect.

Whatever the precise mechanism of transmission of the disease may be there is a great weight of evidence to suggest that the probability of becoming infected varies as the intimacy of contact with an open patient. Patients have been known where the contact has been very brief; however they may be regarded as exceptional. It is clear that one component of the familial factor is due to the increased chance of contact when there is an open patient within the family. The mechanism of the transmission of $M$. leprae is a matter of dispute; nevertheless there is wide agreement that the frequency of effective transmission of the bacilli is far higher than the frequency with which new infections are established. This implied that there is variation in susceptibility. This variation in susceptibility may be genetic, environmental or both. There is a tendency for members of any family to be exposed to similar variations in the environment, such as standard of hygiene and diet; such factors may be of importance in their own right or in as much as they affect other factors such as transmission.
On these grounds alone it would be expected that there is a familial effect.

It seems undeniable that there is a familial effect, and that this is made up of what may be termed an 'environmental component' and a 'contagion component'. These components will be of epidemiological importance for all population units.

Another possible component of the familial effect is genetic. There has been no reasonable attempt to gather the information from which an opinion can be formed as to the possibility of there being a genetic control of susceptibility. Any consideration of this problem is made difficult by reason of the factors that have already been discussed.

Leprosy is made manifest in many forms; these may be placed in two main categories: lepromatous leprosy and tuberculoid leprosy. Lepromatous leprosy is characterised by the presence of large numbers of acid-fast bacilli in the tissues. It is the form that is believed to be contagious. If untreated, extensive deformation occurs and ultimately death. It would appear that in this form of the disease there is no resistance to the presence and activities of the bacilli. Tuberculoid leprosy would appear to be characterised by a hypersensitivity to the bacilli. Very few bacilli are found in the tissues, the lesions and deformation that result are considered to be attributable to the host reaction rather than to the direct activities of the bacilli. This form of the disease is not lethal. New patients may be lepromatous 
or tuberculoid in type or they may enter into a dimorphous or intermediate phase which may develop into one or other of the main forms later.

There are striking differences in the relative incidence of the two main forms in different populations. In negroid peoples about $90 \%$ of leprosy patients are tuberculoid and $10 \%$ lepromatous. This contrasts with $45 \%$ lepromatous and $55 \%$ tuberculoid in Caucasians. This could be explained on the basis of environmental variation, but it is found that expatriate peoples show a similar ratio of the two forms to those of their own race living in their native lands, not to those of the indigenous population, and this holds true whether they are living according to the conditions of their own race or according to the conditions of the indigenous population. This must be attributed to genetic differences in race, and it may be presumed that the racial differences are expression of variation in gene frequencies.

It might be considered that the different forms of the disease are expressions of genetic variation in the bacilli rather than in the human hosts. However, this does not account for the evidence of variation within multi-racial communities; furthermore it is difficult to see how the tuberculoid strain could be maintained at a high frequency since new infections are not started from tuberculoid patients. It might be argued that the environment of the host selects one strain over the other in an infection, and that the tuberculoid strain arises by mutation. This cannot be refuted, but it should be noted that it implies host variation.

I intend to collect data of the incidence of the different forms of the disease in a wide variety of communities in order to check the observations quoted. Particular attention will be devoted to the collection of data from areas where the incidence of leprosy has changed as a result of the establishment of leprosy hospitals, and where there are records covering the period of change.

A small amount of data has been gathered concerning the incidence of leprosy of the 2 types in families. These data relate to a comparison between the frequency of similar types of leprosy in identical twins and in sibs of the same sex.

\begin{tabular}{lccc}
\hline \multicolumn{1}{c}{ Relationship } & Similar & Dissimilar & Total \\
\hline Identical twin & 8 & 0 & 8 \\
Sibs of the same sex & 15 & 8 & 23 \\
\hline
\end{tabular}

These data do not show a significant difference between the identical twins and the sibs, this is probably due to the small size of the sample. This evidence must, however, be regarded as very suggestive of a genetic control over susceptibility to either form of the disease.

It is planned to make a wide-scale survey of the pedigrees of leprosy patients.

It is possible to propose several genetic systems that fit such facts as are known. The ratios predicted from a single locus system are not consistent with the known facts, unless there are multiple alleles. The most probable systems are those with 2 pairs of alleles or gene complexes. Such a system is outlined below:A Wild type

a Non-resistance Locus 1

B Wild type

b Hypersensitive

$\mathrm{A}$ and $\mathrm{B}$ partially dominant

Genotypes Phenotypes

AABB Normal resistance

$\mathrm{AABb} \quad$ Normal resistance

AAbb Tuberculoid

AaBB Normal resistance

$\mathrm{AaBb} \quad$ Normal resistance

Aabb Dimorphous tending to tuberculoid aaBB Lepromatous

$\mathrm{aaBb}$ Dimorphous tending to lepromatous

aabb Dimorphous equal tendency to lepromatous or dimorphous

The frequencies of ' $a$ ' and ' $b$ ' low as compared with the wild type alleles and varying in populations.

This system is, of course, highly speculative but provides a guide for the collection of data.

Any assumption that variation in the relative incidence of the different forms of leprosy between populations is attributable to variation 
in gene frequency implies that different selective forces have operated on the populations. These differences are likely to be highly complex.

In medieval times leprosy was a common disease in Europe. There are now very few parts of Europe where the disease is endemic. This decline in the incidence of leprosy occurred before the introduction of effective drugs. Leprosy has been, and to a lesser extent still is, regarded with abhorrence in Europe. Leprosy patients were strictly segregated and their chances of marriage and parenthood were negligible, consequently genes giving susceptibility to leprosy were in effect sterility genes. If the relevant genes were recessive it can be calculated that the gene frequency would have fallen exponentially. The fall would be linear for a dominant gene with complete penetrance but since the penetrance of a leprosy gene is low and since the penetrance would fall with the decline in gene frequency (since the number of open patients would fall and penetrance depends upon contact with an open patient) a dominant gene would be expected to decline in freuency in a similar way to a recessive gene. It has been shown that when selection against a multifactorial genetic system of recessive genes is relaxed the gene frequency may be expected to rise. It would be expected, therefore, that amongst Europeans there would be a relatively high frequency of genes giving susceptibility to leprosy. This expectation is borne out by observation. There is evidence to suggest that resistance to leprosy confers some degree of susceptibility to tuberculosis and vice versa. Therefore, with the decline of leprosy, genes giving susceptibility to lepromatous leprosy would be selected for and those giving susceptibility to tuberculoid leprosy would be selected against on this basis of their effect on tuberculosis. The result of this would be a higher frequency of lepromatous susceptibility genes and hence of lepromatous leprosy in Europeans as compared with the native populations of leprosy endemic areas. This is borne out by observation.

There is not the same intensity of discrimination against leprosy patients in Africa, though there is some discrimination, particularly against people with lepromatous leprosy. In some communities people with lepromatous leprosy may only be able to marry those similarly afflicted. This enforced positive assortative mating would be expected to intensify the effect of selection against the relevant genes, and it is found that the relative incidence of lepromatous leprosy is low in such communities.

It is clear that the problem of gene frequencies in different populations is not simply a genetic problem but a problem of anthropology and social history also. I, therefore, intend to seek the collaboration of workers in the appropriate disciplines.

The work will in the first instance be restricted to the analysis of data obtained by the circulation of questionnaires to leprosy hospitals in a variety of leprosy endemic areas. Further work will depend upon the results of this analysis.

I wish to thank Dr. R. G. Cochrane, Adviser in Leprosy, Ministry of Health, London, for his interest in these proposals and for his offer of help in gathering the necessary information. I also wish to thank Prof. J. M. Thoday for his encouragement and advice as to the form that the investigations should take.

\section{Proposed Investigations into the Genetics of Physiological Systems in the Mouse}

The majority of characters that are studed in animal genetics are morphological. Morphology may be considered as the expression of physiological processes. However, the physiological variations that underlie morphological variation are usually obscure, so that the chain of causation between gene and character is very incompletely known. It, therefore, seems desirable to investigate the genetic systems controlling those physiologi al processes about which there is much detailed knowledge.

The characteristics that make an animal suitable for genetical experiment are often those that make it unsuitable for physiological work. Thus, though Drosophila melanogaster is eminently suitable for genetic studies, it is not a good subject for experimental physiology. The 
animal that offeres the best compromise between the conflicting demands of the 2 types of experiment is probably the mouse.

There is detailed knowledge of some hormone controlled systems in the mouse. Two systems which appear to be suitable for genetical investigation are the control of water metabolism, and the control of the female sexual cycle.

It is possible to measure an animal's daily intake of water. The ratio of water intake to body weight provides information about the water balance that is being maintained by the animal. It is proposed to select mice for extremes of water balance. Lines of mice with a high water intake per unit body weight and with low water intake per unit body weight will be set up. The selected lines will then be compared in detail, following the methods of genetical analysis developed by Prof. Thoday, and the physiological techniques developed by Prof. Chester Jones.

Techniques have been developed by which it is possible to follow the course of the female sexual cycle of the rat. These techniques are very simple and there is little reason to doubt that they can be adapted for use on the mouse. It is proposed to select lines of mice for variation in the length of the different phases of the cycle. Genetical analysis will then be made on the selected lines. Physiological studies will also be made.

These investigations have not been initiated yet. Even when they are started pilot experiments will be essential and these will take a long time. The project is, therefore, a long-term one and it will be several years before any meaningful results can be expected. 